\title{
Phase IV Study of Bevacizumab in Combination with Infusional Fluorouracil, Leucovorin and Irinotecan (FOLFIRI) in First-Line Metastatic Colorectal Cancer
}

\author{
Alberto Sobrero $^{\mathrm{a}}$ Stephen Ackland $^{\mathrm{b}}$ Stephen Clarke ${ }^{c}$ Ramón Perez-Carrión $^{\mathrm{d}}$ \\ Silvana Chiara ${ }^{\mathrm{e}}$ John Gapski ${ }^{\mathrm{f}}$ Paul Mainwaring ${ }^{g} \quad$ Bernd Langer $^{\text {h }}$ Scott Young ${ }^{\mathrm{i}}$ \\ for the AVIRI Trial investigators \\ ${ }^{a}$ Cliniche Universitarie Convenzionate UO, Genoa, Italy; ${ }^{b}$ Newcastle Mater Misericordiae Hospital, \\ Medical Oncology, and Hunter Medical Research Institute Cancer Research Program, Waratah, N.S.W., and \\ ${ }^{c}$ Concord Repatriation General Hospital, Medical Oncology, Concord, N.S.W., Australia; ${ }^{d}$ Hospital Quiron, \\ Oncologia, Pozuelo de Alarcon, Madrid, Spain; ${ }^{e}$ Istituto Nazionale per la Ricerca sul Cancro, Medical Oncology,

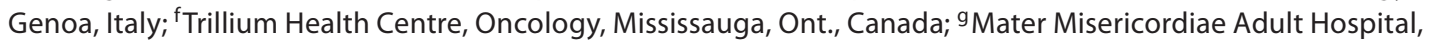 \\ Medical Oncology, South Brisbane, Qld., Australia; hF. Hoffmann-La Roche Ltd., Basel, Switzerland; 'Northeastern \\ Ontario Regional Cancer Centre, Oncology, Sudbury, Ont., Canada
}

\section{Key Words}

Antiangiogenic therapy $\cdot$ Bevacizumab $\cdot$ Colorectal cancer • FOLFIRI $\cdot$ Progression-free survival $\cdot$ Vascular endothelial growth factor

\begin{abstract}
Background: Bevacizumab (Avastin ${ }^{\circledR}$ ) significantly improves overall survival (OS) and progression-free survival (PFS) when combined with first-line irinotecan (IFL) plus bolus 5-fluorouracil (5-FU) and leucovorin (LV) in patients with metastatic colorectal cancer (CRC). This open-label, phase IV trial evaluated the efficacy and safety of first-line bevacizumab in combination with IFL and infusional 5-FU/LV (FOLFIRI). Methods: Two-hundred and nine treatment-naïve metastatic CRC patients were enrolled and received bevacizumab and FOLFIRI every 2 weeks. Treatment was continued
\end{abstract}

until disease progression. The primary objective was PFS, with additional determinations of $O S$, response and toxicity. Results: Median PFS was 11.1 months and is comparable to that observed in published phase III and community-based trials using first-line bevacizumab plus FOLFIRI, and to phase III trials using bevacizumab in combination with bolus 5-FU/ LV plus IFL. Median OS was 22.2 months. Overall response rate was $53.1 \%$ and the disease control rate $85.6 \%$. Most adverse events were grade $1 / 2$ and were manageable. The most common grade $3 / 4$ adverse events ( $\geq 10 \%$ ) were neutropenia, venous thromboembolic events, diarrhea, and fatigue. Conclusion: Bevacizumab combined with first-line FOLFIRI is an effective and well-tolerated therapy option for patients with metastatic CRC.

Copyright $\odot 2009$ S. Karger AG, Basel

\section{KARGER}

Fax +41613061234 E-Mail karger@karger.ch www.karger.com
(C) 2009 S. Karger AG, Basel 0030-2414/09/0772-0113\$26.00/0

Accessible online at: www.karger.com/ocl
Alberto Sobrero

UO di Oncologia Medica, Azienda Ospedaliera Universitaria

San Martino, Lgo Rosanna Benzi, 10

IT-16132 Genoa (Italy)

Tel. +39 010555 3301, Fax +39 010555 5141, E-Mail alberto.sobrero@hsanmartino.it 


\section{Introduction}

Colorectal cancer (CRC) is currently the second most frequently diagnosed cancer, with approximately 410,000 new cases ( $13 \%$ of all new cancer diagnoses) in Europe in 2006. In addition, CRC accounted for 207,000 deaths during the same time period [1]. Approximately $15 \%$ of patients with CRC will present with metastatic disease, and a further $40-50 \%$ will go on to develop metastases during the course of their disease [2]. While recent improvements in chemotherapy regimens have extended survival duration for such patients, chemotherapies alone generally offer $<20$ months survival [3-5]. Thus, new, more effective therapies are required.

Bevacizumab is a humanized monoclonal antibody that inhibits tumor angiogenesis by blocking vascular endothelial growth factor (VEGF), the key mediator of angiogenesis, and is the first antiangiogenic agent approved for the treatment of cancer. A wealth of clinical data are available that show bevacizumab improves overall survival (OS) and/or progression-free survival (PFS) when combined with bolus 5-fluorouracil/leucovorin (5-FU/ LV) [6], bolus 5-FU/LV plus irinotecan (IFL) [7], infusional 5-FU/LV plus oxaliplatin (FOLFOX) $[5,8]$ and capecitabine plus oxaliplatin (CAPOX) [5] in patients with metastatic CRC.

As a consequence, bevacizumab was recently approved by the European Commission for use in combination with fluoropyrimidine-based chemotherapy in patients with metastatic carcinoma of the colon or rectum. Here we report the results from a phase IV, multicenter, nonrandomized, open-label trial of first-line bevacizumab plus IFL and infusional 5-FU/LV (FOLFIRI) in patients with previously untreated metastatic CRC. This study was requested by the regulatory authorities following the initial approval of bevacizumab based on the survivalimprovement in the pivotal trial AVF2107g [7].

\section{Patients and Methods}

\section{Eligible Patients}

Patients with previously untreated, histologically or cytologically confirmed carcinoma of the colon and/or rectum with evidence of metastases were eligible for this study. In addition, patients had: $\geq 18$ years; no prior adjuvant or neo-adjuvant chemotherapy/radiotherapy within 6 months before inclusion; an Eastern Cooperative Oncology Group (ECOG) performance status 0 or 1 , and adequate hematological and liver function. Written informed consent was obtained from each patient prior to the study.
Exclusion criteria included: use of adjuvant IFL or anti-VEGF therapy; prior chemotherapy for metastatic CRC; major surgery within 28 days before trial treatment; ongoing full-dose oral or parenteral anticoagulant or antiplatelet aggregation treatment.

\section{Study Design}

The study was an international, multicenter, open-label, phase IV trial of first-line bevacizumab plus FOLFIRI in patients with metastatic CRC conducted according to ICH-GCP guidelines and approved by properly constituted ethics committees in each jurisdiction. Although standard FOLFIRI was the preferred regimen for this trial, minor violations on the standard regimen according to local standards were allowed.

Patients were treated with bevacizumab $5 \mathrm{mg} / \mathrm{kg}$ on day 1 , IFL $180 \mathrm{mg} / \mathrm{m}^{2}$ on day 1 with infusional $5-\mathrm{FU} / \mathrm{LV}$ (LV $400 \mathrm{mg} / \mathrm{m}^{2}$, equivalent to $200 \mathrm{mg} / \mathrm{m}^{2} \mathrm{~L}$-folinic acid, by infusion on day 1) followed by 5 -FU $400 \mathrm{mg} / \mathrm{m}^{2}$ bolus, then 5 -FU $2,400 \mathrm{mg} / \mathrm{m}^{2}$ as a 46 hour continuous infusion. Therapy was repeated every 2 weeks for a minimum of 6 cycles, after which chemotherapy could be adjusted for individual patients, provided that 5-FU was given as continuous infusion as per protocol. Dose modifications of chemotherapy were prescribed, according to toxicity experienced. Bevacizumab was continued until disease progression, unacceptable toxicity or death. No dose modifications for bevacizumab were permitted except for body weight change of $\pm 10 \%$ from baseline.

The trial was sponsored by F. Hoffmann-La Roche Ltd., Basel, Switzerland.

\section{Efficacy and Safety}

The primary endpoint of PFS was used to evaluate the efficacy of combining bevacizumab with FOLFIRI. Due to the availability of effective second-line treatment options that may confound the efficacy analysis of this study, OS was not used as the primary endpoint. OS, response rate, duration of response and safety were all secondary endpoints of this trial.

Tumor assessments were based on Response Evaluation Criteria in Solid Tumors (RECIST) and performed every 3 months for the first 12 months and every 4 months thereafter. Response was confirmed by a second assessment at least 4 weeks later. Safety assessments were made at the time of chemotherapy administration during the first 12 weeks and every 4 weeks thereafter. Adverse events were graded according to the National Cancer Institute Common Terminology Criteria for Adverse Events version 3.0.

\section{Statistical Considerations}

The sample size $(\mathrm{n}=200)$ was selected to allow comparison of study results with historical controls. The sample size accounted for a dropout rate of $25 \%$, leaving 150 patients for evaluation. Assuming that the median PFS of historical controls was 7 months, an improvement of 2 months would give a 58\% PFS rate at 7 months for patients receiving bevacizumab plus chemotherapy. With 150 evaluable patients, the 2 -sided $95 \%$ confidence interval (95\% CI) for PFS would not include the 7 months' PFS rate of 50\% for historical controls, which would provide evidence for improved PFS. 
Table 1. Patient demographics (ITT and safety populations, $\mathrm{n}=209$ )

\begin{tabular}{|c|c|}
\hline Parameter & \\
\hline \multicolumn{2}{|l|}{ Age, years } \\
\hline Median & 62 \\
\hline Range & $31-82$ \\
\hline Gender: male, $\%$ & 60 \\
\hline Race: Caucasian/Asian/other, \% & $92 / 6 / 2$ \\
\hline \multicolumn{2}{|c|}{ Time since diagnosis of metastatic CRC, months } \\
\hline Median & 2.9 \\
\hline Range & $0.2-162.2$ \\
\hline \multicolumn{2}{|l|}{ Site of disease at first diagnosis } \\
\hline Colon, \% & 67.9 \\
\hline Rectum, \% & 21.5 \\
\hline Colon and rectum, \% & 10.5 \\
\hline \multicolumn{2}{|l|}{ Adjuvant chemotherapy } \\
\hline $5-\mathrm{FU}$ & $58(28 \%)$ \\
\hline Oxaliplatin & $5(2 \%)$ \\
\hline \multicolumn{2}{|c|}{ Time since adjuvant chemotherapy, months } \\
\hline Median & 15.7 \\
\hline Range & $5.7-95.8$ \\
\hline ECOG performance status $0 / 1, \%$ & $62 / 38$ \\
\hline \multicolumn{2}{|c|}{ Predominant sites of metastatic disease at baseline (target lesions) } \\
\hline Liver, \% & 72 \\
\hline Lung/pleura, \% & 23 \\
\hline Lymph nodes, \% & 26 \\
\hline
\end{tabular}

\section{Results}

\section{Patients}

A total of 209 patients were enrolled at 31 centers in Australia, Canada, Italy, Spain and China from April 2005 to November 2005. All these patients were included in the intent-to-treat (ITT) and safety populations. One hundred and ninety-six patients were included in the per-protocol population (PPP); most frequent reasons for exclusion from PPP being absence of baseline and/or postbaseline tumor assessment (7 patients) and termination of study treatment before 12 weeks for reasons other than disease progression or death (6 patients). Patient demographics for the ITT population are shown in table 1.

At the time of the clinical cut-off for the follow-up analysis (31 October 2007), 117 patients had died, 84 were alive in the follow-up phase and 8 patients were alive on treatment. The median duration of follow-up was 21.7 months (range 0.8-29.7).

One-hundred and fifty-six (75\%) of the enrolled patients were treated with standard FOLFIRI (every 2 weeks)
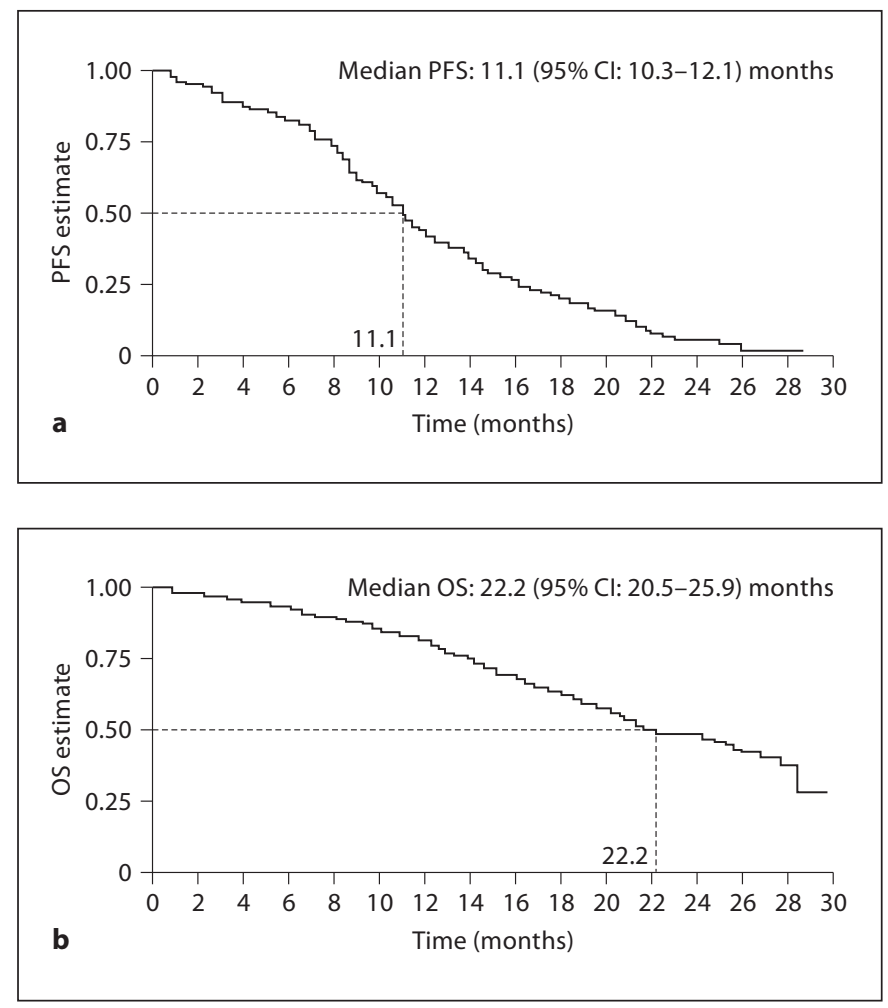

Fig. 1. ITT population. a Kaplan-Meier plots of PFS. b KaplanMeier plot of OS.

as per protocol and 53 patients $(25 \%)$ received FOLFIRI modified according to local practice.

\section{Duration of Treatment}

All 209 patients were treated with at least 1 dose of bevacizumab, $89 \%$ received at least 6 administrations and $45 \%$ received 20 or more administrations of bevacizumab treatment. The median duration of bevacizumab treatment was 9.0 months (275 days). The median duration of chemotherapy (IFL, 5-FU, folinic acid) ranged from 6.4 to 6.5 months (196-199 days).

\section{Efficacy}

Efficacy was similar in patients who received modified FOLFIRI and those who received the standard FOLFIRI regimen. The median duration of PFS was 11.1 months (337 days; 95\% CI: 10.3-12.1 months) in the ITT population (fig. 1a). The survival estimate at 6 months was $94 \%$ (95\% CI: $91-97 \%$ ) and $82 \%$ (95\% CI: $77-87 \%$ ) at 12 months. Median OS was 22.2 months (676 days; 95\% CI: 20.5-25.9 months) in the ITT population (fig. 1b). The addition of bevacizumab to FOLFIRI led to a confirmed response in 
Table 2. Summary of grade $3 / 4$ adverse events $(n=209)$

\begin{tabular}{lc}
\hline Parameter & Patients, \% \\
\hline Neutropenia & 29 \\
Venous thromboembolic events $^{1}$ & $18^{2}$ \\
Diarrhea & 12 \\
Fatigue & 10 \\
Vomiting & 7 \\
Deep vein thrombosis & 7 \\
Pulmonary embolism & $7^{2}$ \\
Nausea & 6 \\
Febrile neutropenia $^{2}$ & $6^{2}$ \\
Hypertension $^{1}$ & 5 \\
Bleeding $^{1}$ & 4 \\
Arterial thromboembolic events $^{1}$ & $4^{2}$ \\
Proteinuria $^{1}$ & 2 \\
Gastrointestinal perforation $^{1}$ & $2^{2}$ \\
Wound-healing complications $^{1}$ & $<1$ \\
Congestive heart failure $^{1}$ & 1 \\
Fistula/abscess $^{1}$ & $1^{2}$ \\
\hline
\end{tabular}

1 'Targeted' adverse events considered possibly or probably related to Avastin treatment based on previous clinical studies.

${ }^{2}$ Including 1 event leading to death.

$111 / 209$ patients (53.1\%) of whom $8(3.8 \%)$ had a complete response and $103(49.3 \%)$ had a partial response. A further 68 patients (32.5\%) had stable disease, resulting in a disease control rate of $85.6 \%$ at week 12 . Sixteen patients (7.7\%) had no confirmed response or immediate progressive disease before the first scheduled tumor assessment, and response assessments were not available for $14 \mathrm{pa}-$ tients (6.7\%). The median time from first study treatment to first confirmed response was 4.5 months (137 days; 95\% CI: 3.2-5.3 months) and the median duration of response was 9.0 months (274 days; 95\% CI: 8.1-11.3 months). A similar efficacy profile was observed in the PPP (data not shown).

\section{Safety}

Overall, bevacizumab in combination with FOLFIRI was generally well tolerated, with no new safety concerns reported. The majority of adverse events were grade $1 / 2$; the incidences of the most common grade $3 / 4$ adverse events are summarized in table 2 . Table 2 also highlights those 'targeted' adverse events that historically are considered to be probably or possibly related to bevacizumab based on previous bevacizumab clinical trials (35\% of patients experienced at least 1 grade $3 / 4$ 'targeted' adverse event). Fifty-three per cent of patients experienced bleed- ing events, mostly epistaxis, but only $4 \%$ of bleeding events were grade 3 . Hypertensive events occurred in $28 \%$ of patients, of whom only $5 \%$ had grade 3 hypertension. Bevacizumab was discontinued in 2 patients who experienced hypertension; however, the majority of patients $(76 \%)$ were treated for hypertension, with resolution in most cases (76\%). A total of 7 patients experienced proteinuria, with only 4 having a grade 3 event. Nephrotic syndrome was not observed. Venous thromboembolic events (VTEs) were observed in $24 \%$ of all patients and $18 \%$ of all patients had grade $3 / 4$ VTEs (with 1 event leading to death). Seventeen patients underwent surgery during treatment and of these patients only 1 patient had wound-healing complications. Alopecia was observed in $46 \%$ of the patients.

Adverse events leading to discontinuation of any component of the study treatment regimen occurred in $24 \%$ of patients; gastrointestinal disorders were the most common events leading to discontinuation. A total of 42 patients (20\%) withdrew from bevacizumab treatment due to an adverse event, with pulmonary embolism (3\%) being the most frequent individual event leading to bevacizumab withdrawal. A similar number of patients ( $\mathrm{n}=$ $41,20 \%$ ) withdrew from chemotherapy due to an adverse event, with diarrhea (2\%) being the most frequent individual event leading to chemotherapy withdrawal. Adverse events leading to dose modification or treatment interruption of any component occurred in $79 \%$ of patients and $61 \%$ of the patients missed at least 1 dose of bevacizumab. Adverse events leading to modification of chemotherapy occurred in $77 \%$ of patients; the major reasons were neutropenia (42\%), diarrhea (13\%), mucositis (8\%), febrile neutropenia (5\%) and fatigue (5\%).

Throughout the study (duration 2 years and 7 months), a total of 117 deaths (56\%) were reported, the majority ( $n=110,94 \%)$ of which were due to progressive disease. Of the remaining 7 deaths $(6.0 \%$; $3.4 \%$ of total population), 5 were attributed to adverse events, including pulmonary embolism, myocardial infarction, anal fistula, aortic dissection, cardiac arrest, hepatic failure, large-intestinal perforation, neutropenic sepsis, small-intestinal obstruction and tumor hemorrhage; the cause of death in 2 patients was reported as not known. During the treatment phase until disease progression, including the standard study observation period (28 days safety follow-up), 9 deaths were reported; 5 were a direct result of the patients' disease and 4 were related to adverse events, resulting in an adverse-event related mortality rate of $1.9 \%$. The 60 -day mortality rate was $2 \%$. 


\section{Discussion}

Having a relatively short plasma half-life, 5-FU administered by bolus injection is quickly cleared from the blood whereas infusional administration prolongs 5-FU exposure [9]. As such, several studies have demonstrated superior efficacy of infusional 5-FU over bolus 5-FU, with reduced toxicity [10-12]. Based on these data, it is conceivable that the addition of bevacizumab to FOLFIRI may be a more effective and tolerable combination regimen for the treatment of this disease than the combination of bevacizumab and IFL/5-FU bolus regimens.

Results from this phase IV trial support the use of bevacizumab in combination with FOLFIRI for the first-line treatment of patients with metastatic CRC. Treatment with this combination regimen resulted in an overall response rate of $53.1 \%$, which is favorable in comparison to the $44.8 \%$ reported in patients treated with bevacizumab plus IFL in the pivotal AVF2107g trial [7]. In terms of survival, patients treated with bevacizumab plus FOLFIRI in the present trial had a median PFS of 11.1 months, which is comparable to the 10.6 months achieved in AVF2107g [7] and the PFS of 11.2 months recently published for the randomized phase III BICC-C trial [13]. The BICC-C trial was initially designed to evaluate modified IFL (mIFL), FOLFIRI or capecitabine plus IFL (CAPIRI) with or without celecoxib in 430 patients. The trial was later amended to evaluate FOLFIRI or mIFL with or without bevacizumab in 117 patients. Addition of bevacizumab to each of the chemotherapy regimens resulted in improved PFS of 11.2 months in the FOLFIRI arm (vs. 7.6 months for FOLFIRI alone) and 8.3 months in the mIFL arm (vs. 5.9 months for mIFL alone) [13]. The near identical PFS improvements in the current trial and BICC-C trial provide further evidence that patients with CRC can have a long progression-free interval with first-line chemotherapy when combined with bevacizumab. Further support for the use of bevacizumab in combination with FOLFIRI comes from a single-arm phase II trial in which 43 patients had a median PFS of 12.5 months and 1-year survival rate of 95\% [14]. These results are encouraging as they are equivalent, if not better, than the results obtained from phase II and III trials $[7,15]$ - historically, clinical results from earlier trials are usually better as they have a more selected patient base. In addition, these studies clearly demonstrate that bevacizumab is effective with an improved IFL protocol, i.e. FOLFIRI.

Bevacizumab plus FOLFIRI in First-Line

Metastatic Colorectal Cancer
PFS data from AVF2107g and the present trial are comparable ( $\sim 11$ months). Replacing the 5 -FU part of the regimen with capecitabine (CAPIRI) results in a similar, if not longer, median PFS as reported in the AIO 0604 trial [16]. These data are also consistent with those reported in the randomized phase III trials PACCE [17] and CAIRO-2 [18], in which bevacizumab was combined with oxaliplatin-based chemotherapy. However, these data are slightly higher than the 9.4 months, PFS reported for another randomized phase III trial of bevacizumab combined with oxaliplatin-based chemotherapy (NO16966) [5]. In order to provide a possible explanation for the difference, we examined AVF2107g and the present trial in which patients continued to receive bevacizumab for about 2-3 months after discontinuation of chemotherapy. In contrast, bevacizumab was discontinued in patients at the same time as chemotherapy ( $\sim 6$ months) in NO16966, impacting on PFS. These observations suggest that maintaining bevacizumab therapy, at least until disease progression, may be important for optimal clinical benefit.

The efficacy of bevacizumab in combination with FOLFIRI reported from clinical trials has also been reproduced in the community setting. Two observational studies, BRiTE and BEAT, were designed to evaluate the safety and efficacy of bevacizumab in large, less-selected, community-based patient populations $[19,20]$. Patients treated with bevacizumab plus FOLFIRI achieved a median PFS of 10.9 months (95\% CI: 9.7-11.8) in the BRiTE study [21] and 11.6 months in the BEAT study [20]. In the BEAT study, the median OS for patients receiving bevacizumab plus FOLFIRI was 23.7 months compared with 22.7 months for the total patient population [20]. Thus, the clinical benefit of bevacizumab plus FOLFIRI is consistent across studies and settings.

In the current study, bevacizumab in combination with FOLFIRI was well tolerated; however, although no new safety signals were reported, the occurrence of rare, but severe, adverse events attributable to bevacizumab was confirmed. Mortality due to adverse events was in a similar range as in the pivotal phase III trial AVF2107g [7]. The incidence of all-grade arterial thromboembolic events (4.3\%) in the current study was similar to that observed in the pivotal phase III trial (3.3\%) (AVF2107g Clinical Study Report); however, it was somewhat higher than that observed in trials involving oxaliplatin-based chemotherapy [8] or 5-FU-based chemotherapy in a less selected patient population $[19,20]$. IFL itself appears to be associated with an increased risk of thrombosis [22], which may explain the findings within this study. An-

Oncology 2009;77:113-119 
other reason for the apparent increased incidence of VTEs, when compared to AVF2107g, might be the use of different coding tools (COSTART for AVF2107g vs. MedDRA for MO18458 [AVIRI]).

Extensive data are available for the safety profile of bevacizumab, with over 250,000 cancer patients having been treated [23]; 4,000 of these patients coming from observational studies in metastatic CRC (BRiTE [19] and BEAT [20]). Bevacizumab is generally well tolerated in combination with standard chemotherapy regimens, and adverse events are mostly mild to moderate in severity and manageable using standard techniques $[5-8,19,20]$. It is important to note that the clinical benefit of adding bevacizumab to chemotherapy means that patients receive prolonged treatment with increased treatment exposure. However, this is associated with minimal or no increase in toxicity.
In conclusion, antiangiogenic therapy with bevacizumab plus FOLFIRI is an effective and well-tolerated regimen for the first-line treatment of metastatic CRC, and appears to be equally effective in clinical trials and community-based settings. Available data suggest that bevacizumab, combined with FOLFIRI or any fluoropyrimidine-containing chemotherapy regimen, should be considered as the therapy of choice for the treatment of patients with metastatic CRC.

\section{Acknowledgements}

Medical writing support was provided by Tawhid Ahmad of Gardiner-Caldwell Communications. This study and medical writing support were funded by F. Hoffmann-La Roche Ltd. Alberto Sobrero is a member of the advisory board for F. Hoffmann-La Roche Ltd. and has participated in F. HoffmannLa Roche Ltd. satellite symposia.

\section{References}

$>1$ Ferlay J, Autier P, Boniol M, Heanue M, Colombet M, Boyle P: Estimates of the cancer incidence and mortality in Europe in 2006. Ann Oncol 2007;18:581-592.

$\checkmark 2$ Van Cutsem E, Nordlinger B, Adam R, Köhne CH, Pozzo C, Poston G, Ychou M, Rougier P; European Colorectal Metastases Treatment Group: Towards a pan-European consensus on the treatment of patients with colorectal liver metastases. Eur J Cancer 2006;42:2212-2221.

3 Douillard JY, Sobrero A, Carnaghi C, Comella P, Díaz-Rubio E, Santoro A, Van Cutsem E: Metastatic colorectal cancer: integrating irinotecan into combination and sequential chemotherapy. Ann Oncol 2003; 14(suppl 2):ii7-ii12.

4 Goldberg RM, Sargent DJ, Morton RF, Fuchs CS, Ramanathan RK, Williamson SK, Findlay BP, Pitot HC, Alberts S: Randomized controlled trial of reduced-dose bolus fluorouracil plus leucovorin and irinotecan or infused fluorouracil plus leucovorin and oxaliplatin in patients with previously untreated metastatic colorectal cancer: a North American Intergroup Trial. J Clin Oncol 2006;24:3347-3353.

5 Saltz L, Clarke S, Díaz-Rubio E, Scheithauer W, Figer A, Wong R, Koski S, Lichinitser M, Yang TS, Rivera F, Couture F, Sirzén F, Cassidy J: Bevacizumab in combination with oxaliplatin-based chemotherapy as first-line therapy in metastatic colorectal cancer: a randomized phase III study. J Clin Oncol 2008;26:2013-2019.
6 Kabbinavar FF, Hambleton J, Mass RD, Hurwitz HI, Bergsland E, Sarkar S: Combined analysis of efficacy: the addition of bevacizumab to fluorouracil/leucovorin improves survival for patients with metastatic colorectal cancer. J Clin Oncol 2005;23: 3706-3712.

7 Hurwitz H, Fehrenbacher L, Novotny W, Cartwright T, Hainsworth J, Heim W, Berlin J, Baron A, Griffing S, Holmgren E, Ferrara N, Fyfe G, Rogers B, Ross R, Kabbinavar F: Bevacizumab plus irinotecan, fluorouracil, and leucovorin for metastatic colorectal cancer. N Engl J Med 2004;350:2335-2342.

$\checkmark 8$ Giantonio BJ, Catalano PJ, Meropol NJ, O'Dwyer PJ, Mitchell EP, Alberts SR, Schwartz MA, Benson AB 3rd; Eastern Cooperative Oncology Group Study E3200: Bevacizumab in combination with oxaliplatin, fluorouracil, and leucovorin (FOLFOX4) for previously treated metastatic colorectal cancer: results from the Eastern Cooperative Oncology Group Study E3200. J Clin Oncol 2007;25:1539-1544.

$\checkmark 9$ Vogelzang NJ: Continuous infusion chemotherapy: a critical review. J Clin Oncol 1984; 2:1289-1304.

10 Lokich JJ, Ahlgren JD, Gullo JJ, Philips JA, Fryer JG: A prospective randomized comparison of continuous infusion fluorouracil with a conventional bolus schedule in metastatic colorectal carcinoma: a Mid-Atlantic Oncology Program Study. J Clin Oncol 1989; 7:425-432.
11 Hansen RM, Ryan L, Anderson T, Krzywda B, Quebbeman E, Benson A 3rd, Haller DG, Tormey DC: Phase III study of bolus versus infusion fluorouracil with or without cisplatin in advanced colorectal cancer. J Natl Cancer Inst 1996;88:668-674.

$\checkmark 12$ Efficacy of intravenous continuous infusion of fluorouracil compared with bolus administration in advanced colorectal cancer: Meta-analysis Group in Cancer. J Clin Oncol 1998;16:301-308.

13 Fuchs CS, Marshall J, Mitchell E, Wierzbicki R, Ganju V, Jeffery M, Schulz J, Richards D, Soufi-Mahjoubi R, Wang B, Barrueco J: Randomized, controlled trial of irinotecan plus infusional, bolus, or oral fluoropyrimidines in first-line treatment of metastatic colorectal cancer: results from the BICC-C Study. J Clin Oncol 2007;25:4779-4786.

14 Kopetz S, Glover K, Eng C, Wolff RA, Chang DZ, Adinin RB, Morris J, Abbruzzese JL, Hoff PM: Phase II study of infusional 5-fluorouracil, leucovorin, and irinotecan (FOLFIRI) plus bevacizumab as first-line treatment for metastatic colorectal cancer (abstract 4089). J Clin Oncol 2007;25(suppl):185s.

15 Giantonio BJ, Levy DE, O’Dwyer PJ, Meropol NJ, Catalano PJ, Benson AB 3rd; Eastern Cooperative Oncology Group: A phase II study of high-dose bevacizumab in combination with irinotecan, 5-fluorouracil, leucovorin, as initial therapy for advanced colorectal cancer: results from the Eastern Cooperative Oncology Group study E2200. Ann Oncol 2006;17:1399-1403. 
16 Reinacher-Schick AC, Kubicka S, Freier W, Arnold D, Dietrich G, Geissler M, Hegewisch-Becker S, Graeven U, Schmoll H, Schmiegel W: The combination of bevacizumab (Bev) with capecitabine/irinotecan (CapIri/Bev) or capecitabine/oxaliplatin (CapOx/Bev) is highly active in advanced colorectal cancer (ACRC): a randomized phase II study of the AIO Colorectal Study Group (AIO trial 0604) (abstract 4030). J Clin Oncol 2008;26(suppl):185s.

17 Hecht JR, Mitchell E, Chidiac T, Scroggin C, Hagenstad C, Spigel D, Marshall J, Cohn A, Shahin S, Griffin T: Gastrointestinal Cancers Symposium, Orlando, January 2008, (abstract 273). Available at: http://www.asco. org. Accessed 8 May 2008.
18 Punt CJ, Tol J, Rodenburg JC, Cats A, Creemers G, Schrama JG, Erdkamp FL, Vos A, Mol L, Antonini NF: Randomized phase III study of capecitabine, oxaliplatin, and bevacizu-mab with or without cetuximab in advanced colorectal cancer (ACC), the CAIRO2 study of the Dutch Colorectal Cancer Group (DCCG) (abstract LBA4011). J Clin Oncol 2008;26(suppl):1008s.

19 Kozloff MF, Sugrue MM, Purdie DM, Berlin JD, Flynn PJ, Kabbinavar FF, Sargent DJ, Dong W, Grothey A: Safety and effectiveness of bevacizumab (BV) and chemotherapy (CT) in elderly patients (pts) with metastatic colorectal cancer (mCRC): results from the BRiTE observational cohort study (abstract 4026). J Clin Oncol 2008;26(suppl):184s.

20 Van Cutsem E, Berry S, Michael M, Kretzschmar A, Rivera F, DiBartolomeo M, Mazier M, André N, Cunningham D: Safety and efficacy of bevacizumab plus standard first-line chemotherapy in patients with metastatic colorectal cancer First BEAT (abstract 357P). Ann Oncol 2008;19(suppl): viii25.
21 Kozloff M, Hainsworth J, Badarinath S, Flynn PJ, Kabbinavar F, Sargent D, Purdie DM, Dong W, Grothey A: Safety and effectiveness of bevacizumab (BV) and chemotherapy (CT) in elderly patients (pts) with metastatic colorectal cancer (mCRC): results from the BRiTE Prospective Cohort Study (abstract 375). 2007 Gastrointestinal Cancers Symposium, Orlando, January 2007. Available at: http://www.asco.org. Accessed 8 May 2008.

22 Bleiberg H, Leo AD: Mortality associated with irinotecan plus bolus fluorouracil/leucovorin. J Clin Oncol 2002;20:1144-1149.

23 F. Hoffmann-La Roche Ltd.: data on file. February 2008 


\section{Erratum}

In the article entitled 'Phase IV Study of Bevacizumab in Combination with Infusional Fluorouracil, Leucovorin and Irinotecan (FOLFIRI) in First-Line Metastatic Colorectal Cancer' by Sobero et al. (Oncology 2009;77:113-119) the name Silvia Chiara should read Silvana Chiara. 